OPEN ACCESS

Edited by:

Sujogya Kumar Panda, KU Leuven, Belgium

Reviewed by:

Evandro Leite de Souza, Federal University of Paraíba, Brazil

Debasmita Dubey,

Sambalpur University, India

${ }^{*}$ Correspondence:

Yong Wu

wuyong_zn@csu.edu.cn

Yuan Wu

korchim@126.com

Specialty section:

This article was submitted to Microbial Physiology and Metabolism,

a section of the journal

Frontiers in Microbiology

Received: 23 February 2020

Accepted: 07 October 2020

Published: 02 November 2020

Citation:

Luo Z, Yue S, Chen T, She P, Wu Y and Wu Y (2020) Reduced Growth of Staphylococcus aureus Under High Glucose Conditions Is Associated With Decreased

Pentaglycine Expression.

Front. Microbiol. 11:537290. doi: 10.3389/fmicb.2020.537290

\section{Reduced Growth of Staphylococcus aureus Under High Glucose Conditions Is Associated With Decreased Pentaglycine Expression}

\author{
Zhen Luo ${ }^{1}$, Shan Yue ${ }^{2}, \mathrm{Ti}$ Chen ${ }^{1}$, Pengfei She ${ }^{1}$, Yuan $W u^{1 *}$ and Yong $W^{1 *}$ \\ ' Department of Laboratory Medicine, The Third Xiangya Hospital, Central South University, Changsha, China, ${ }^{2}$ Department \\ of Laboratory Medicine, Hunan Normal University School of Medicine, Changsha, China
}

The high-glucose-induced cytotoxicity in diabetes has been widely recognized. Staphylococcus aureus is the most frequent pathogen isolated from diabetic foot ulcers, but the properties of this bacterium under high glucose conditions remain unclear. S. aureus grew in medium usually forms weak biofilm, and which was significantly increased by addition of glucose. However, extracellular DNA (eDNA), an important component of biofilms, was markedly decreased in presence of $15 \mathrm{mM}$ glucose. The reduced eDNA content was not caused by degradation, because the nuclease activity of biofilm supernatants with glucose was significantly decreased due to the acidic $\mathrm{pH}$ of the medium. Under planktonic state, the growth of $S$. aureus was significantly decreased in the Luria-Bertani (LB) medium supplemented with $25 \mathrm{mM}$ glucose, and the reduced growth of $S$. aureus by glucose was dose-dependent. Except for glucose, the growth of planktonic $S$. aureus was also markedly decreased by fructose or sucrose. Amounts of acid metabolites were produced under high glucose conditions, but the survival of planktonic S. aureus was unaffected by these acidic conditions. Cells of $S$. aureus from the culture medium with glucose had a thinner cell wall and highly resistant to lysostaphin compared with the bacteria cultured in LB medium. mRNA expression of genes encoding pentaglycine bridges, the substrate of lysostaphin, was significantly decreased in S. aureus by glucose. In addition to $S$. aureus, the growth of Staphylococcus haemolyticus and Staphylococcus epidermidis was also significantly decreased by an excess of glucose, but strains of Enterococcus faecalis, Escherichia coli, and Pseudomonas aeruginosa were unaffected by glucose. In conclusion, the reduced growth of $S$. aureus under high glucose conditions is due to impairment of the unique cell-wall structure, pentaglycine bridges.

\section{Keywords: Staphylococcus aureus, glucose, eDNA, pentaglycine, lysostaphin resistance}

Abbreviations: GyrB, gyrase B; MIC, minimum inhibitory concentration; TEM, transmission electron microscopy; eDNA, extracellular DNA; gDNA, genomic DNA; CFUs, colony-forming units; PBS, phosphate-buffered saline; PIA, polysaccharide intercellular adhesin; TSB, tryptic soy broth; MALDI-TOF MS, matrix-assisted laser desorption/ionization time-of-flight mass spectrometry; BA, blood agar; LB, Luria-Bertani; 2-DG, 2-deoxy-D-glucose; SD, standard deviation; femABX, factor essential for methicillin resistance ABX; OD, optical density; mRNA, messenger RNA; PCR, polymerase chain reaction; LIA, L-form induced agar. 


\section{INTRODUCTION}

Glucose serves as an important carbohydrate for the growth of Staphylococcus aureus. Carbohydrates support the growth of S. aureus under anaerobic conditions and high nitric oxide stress (Richardson et al., 2008; Vitko et al., 2015). In the skin and softtissue infections model, glucose transporters contribute to the growth of $S$. aureus, which favors the fermentation of glucose over other carbohydrates (Vitko et al., 2016). Our previous study has revealed that fermentation of glucose contributes to aggregation of S. aureus (Luo et al., 2019). These data indicate that glucose is essential for the growth of $S$. aureus. However, high glucose usually induces endothelial and renal cytotoxicity in diabetic patients (Gao et al., 2015; Yi et al., 2018; Liu et al., 2020). S. aureus is the most frequent pathogen isolated from diabetic foot ulcers, but the properties of this bacterium under high glucose conditions remain unclear.

Staphylococcus aureus grew in medium usually forms weak biofilm, and addition of glucose to growth medium is a common practice to simulate biofilm formation in vitro (You et al., 2014). The matrix within biofilm often consists of polysaccharide intercellular adhesin (PIA). However, surface proteins, such as protein A, promote biofilm development in the absence of PIA exopolysaccharide (Merino et al., 2009). Protein A is a major component of the cell wall of $S$. aureus and is suppressed in tryptic soy broth (TSB) medium containing an excess of glucose (Yamada and Matsumoto, 1986). In addition, glucose enhances the killing efficiency of daptomycin against $S$. aureus, which is independent of the proton motive force and unaffected by physiological changes in $\mathrm{pH}$ (Prax et al., 2016). Daptomycin is a lipopeptide antimicrobial agent that hinders peptidoglycan synthesis in the bacterial cell wall. Daptomycin-resistant S. aureus are usually associated with thickening of the cell wall (Bertsche et al., 2011; Bayer et al., 2013). Therefore, glucose is known to be involved in cell-wall synthesis in S. aureus.

The major structural component of the cell wall of S. aureus is peptidoglycan, which is cross-linked by pentaglycine bridges that help maintain the integrity of the cell wall (Labischinski, 1992; Monteiro et al., 2019). Inhibition of pentaglycine bridge formation leads to a substantial decrease in peptidoglycan cross-links and renders the bacteria highly resistant to lysostaphin (Rohrer et al., 1999). Lysostaphin is a glycylglycine endopeptidase secreted by Staphylococcus simulans. Lysostaphin cleaves pentaglycine bridges in staphylococci, thereby hydrolyzing the cell wall and lysing the bacteria (Grundling and Schneewind, 2006; Francius et al., 2008). Several studies have demonstrated that $S$. aureus is susceptible to lysostaphin in vitro and in vivo, indicating that lysostaphin is a promising candidate for treating $S$. aureus infections (Wu et al., 2003; Oluola et al., 2007; Yang et al., 2007; Chen et al., 2014). However, a recent study reported that S. aureus was highly resistant to lysostaphin in nutrient-rich TSB medium (Wu et al., 2019). As glucose is the most important carbon source for $S$. aureus, we hypothesized whether glucose contributes to the lysostaphin resistance observed in $S$. aureus cultured in nutrient-rich medium. Lysostaphin-resistant strains usually exhibit reduced logarithmic growth rate and increased susceptibility to elevated temperatures (Kusuma et al., 2007). Therefore, we speculate that high glucose reduces the expression of pentaglycine, resulted in impaired growth of S. aureus.

\section{MATERIALS AND METHODS}

\section{Bacterial Strains and Culture Conditions}

Eight clinical strains of $S$. aureus and one clinical strain of Staphylococcus haemolyticus (SH01) were isolated from patients with diabetic foot ulcers in the Department of Laboratory Medicine, Third Xiangya Hospital, Central South University, Hunan, China. All clinical isolates were identified by matrix-assisted laser desorption/ionization time-of-flight mass spectrometry (MALDI-TOF MS; Bruker Biotyper, Germany), and the identification scores were all above 2.0. S. aureus Newman was kindly provided by Professor Min Li (Department of Laboratory Medicine, Renji Hospital, Shanghai Jiao Tong University, China), Staphylococcus epidermidis RP62A was supplied by Professor Di Qu (Fudan University, Shanghai, China), and Enterococcus faecalis ATCC29212, Escherichia coli ATCC25922, and Pseudomonas aeruginosa ATCC27853 were kindly provided by Juncai Luo (Hunan Changsha Tiandiren Biotech Co., Ltd., Changsha, China). Bacteria were routinely cultured at $37^{\circ} \mathrm{C}$ on $5 \%$ sheep blood agar (BA) plates (Caring Trading Co., Ltd., Guangdong, China).

\section{CFU Assays}

To determine the growth and survival of bacteria (S. aureus, S. haemolyticus, S. epidermidis, E. faecalis, E. coli, and $P$. aeruginosa) in the presence of sugar, overnight cultures grown in Luria-Bertani (LB) medium at $37^{\circ} \mathrm{C}$ were diluted 1:100 into fresh LB medium supplemented with $25 \mathrm{mM}$ glucose (Sigma), $25 \mathrm{mM}$ fructose (Sigma), or $12.5 \mathrm{mM}$ sucrose (Sigma). Dosedependent growth of $S$. aureus in glucose was assessed by culturing S. aureus Newman in fresh LB medium supplemented with $0,5,15$, and $25 \mathrm{mM}$ glucose. All cultures were incubated at $37^{\circ} \mathrm{C}$ with shaking at $180 \mathrm{rpm}$, and the colony-forming units (CFUs) were determined after 24 and/or $48 \mathrm{~h}$ by plating $5 \mu \mathrm{L}$ serial dilutions on $\mathrm{BA}$, incubating overnight at $37^{\circ} \mathrm{C}$, and enumerating the number of colonies.

\section{Analysis of eDNA by Quantitative PCR}

Extracellular DNA (eDNA) within biofilms was examined by using quantitative polymerase chain reaction (PCR) targeting the gene gyrB (Dengler et al., 2015). Briefly, overnight cultures of S. aureus Newman grown in $\mathrm{LB}$ medium at $37^{\circ} \mathrm{C}$ were diluted 1:100 into fresh LB medium containing $0,1,3,5$, or $15 \mathrm{mM}$ glucose and $6 \mathrm{~mL}$ of these suspensions were cultured in 6well flat bottom polystyrene plates (Corning, China) at $37^{\circ} \mathrm{C}$ for $18 \mathrm{~h}$. The supernatants were carefully removed, and the biofilms were harvested, resuspended in $500 \mu \mathrm{L}$ TE buffer, and mixed at $3000 \mathrm{rpm}$ for 5 min with Mixmate (Eppendorf, Germany). A $10 \mu \mathrm{l}$ aliquot was removed for bacterial numeration. After centrifugation at $12,000 \mathrm{~g}$ for $5 \mathrm{~min}$, the supernatant was passed through a $0.22-\mu \mathrm{m}$ pore-size filter (Millipore), then eDNA in the filtered supernatants was purified using a nucleic acid extraction 

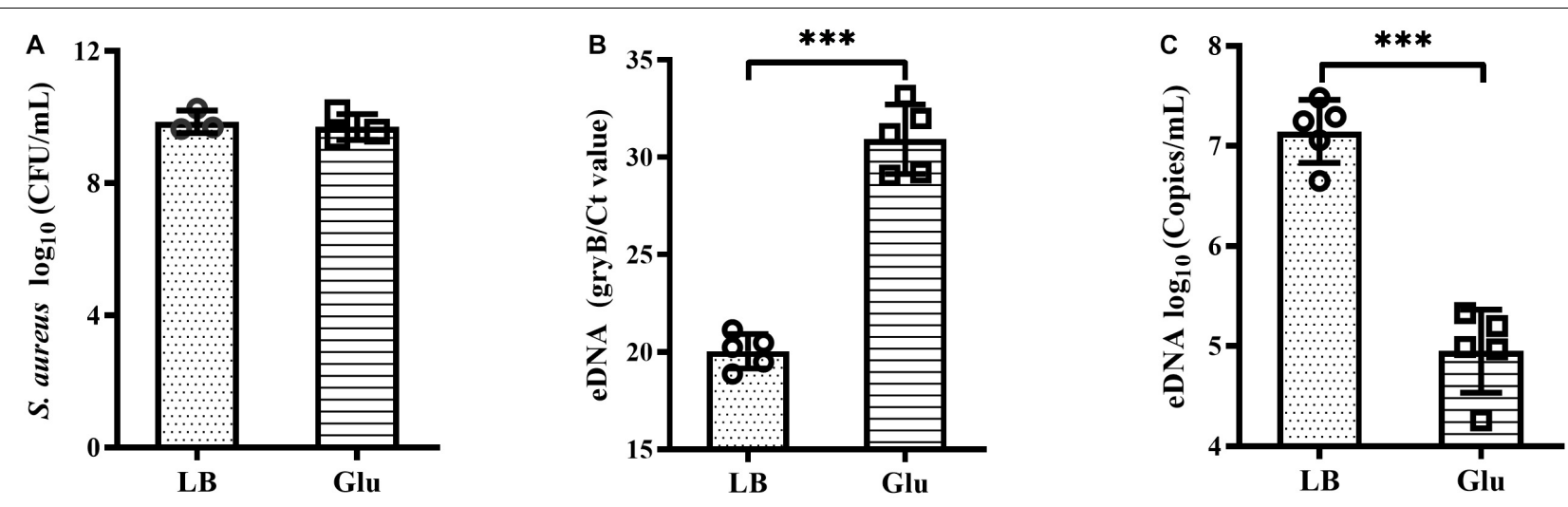

D

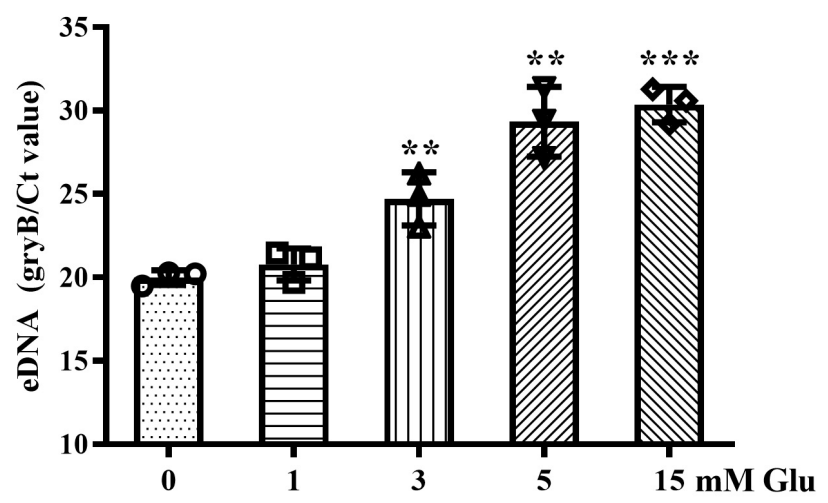

E

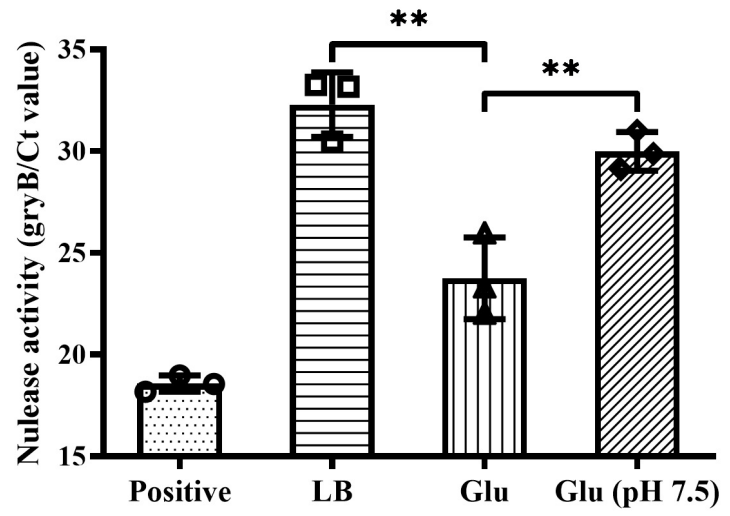

FIGURE 1 | Glucose reduced eDNA levels of S. aureus biofilms. (A) CFUs of biofilms of S. aureus Newman cultured in LB medium with or without 15 mM glucose for $18 \mathrm{~h}$. Supernatants were carefully removed, biofilms were harvested by resuspended in $500 \mu \mathrm{L}$ TE buffer, and the CFUs of the biofilms were determined. (B) eDNA of biofilms quantified by real-time PCR targeting the gene gyrB. (C) eDNA of biofilms quantified using a commercialized PCR-based detection kit. (D) eDNA of biofilms of $S$. aureus Newman cultured in LB medium supplemented with 0, 1, 3, 5, or 15 mM glucose for 18 h. (E) Quantification of gDNA remaining after co-culture of gDNA solution with biofilm supernatants for $30 \mathrm{~min}$; gDNA was quantified by real-time PCR targeting $g y r B$. ${ }^{* *} p<0.01$; ${ }^{* * *} p<0.001$.

kit (Da An Gene Co., Ltd., Sun Yat-sen University, China). The eDNA content was quantified by real-time PCR using the following conditions: pre-denaturation at $94^{\circ} \mathrm{C}$ for $2 \mathrm{~min} ; 40$ cycles of denaturation at $94^{\circ} \mathrm{C}$ for $15 \mathrm{~s}$, annealing at $55^{\circ} \mathrm{C}$ for $20 \mathrm{~s}$, and elongation at $72^{\circ} \mathrm{C}$ for $20 \mathrm{~s}$. Primers used for PCR are shown in the Supplementary Table 1. The eDNA content was also examined with a commercialized PCR-based molecular methicillin-resistant $S$. aureus (MRSA) detection kit (Liferiver, China) according to manufacturer's instructions.

\section{Nuclease Activity Assays}

Overnight cultures of $S$. aureus Newman grown in LB medium at $37^{\circ} \mathrm{C}$ were diluted $1: 100$ into fresh $\mathrm{LB}$ medium with or without $15 \mathrm{mM}$ glucose, and $6 \mathrm{~mL}$ of these suspensions were cultured in 6-well flat bottom polystyrene plates at $37^{\circ} \mathrm{C}$ for $18 \mathrm{~h}$. The supernatants were collected, centrifuged at $3000 \mathrm{rpm}$ for $10 \mathrm{~min}$, the resulting supernatant was passed through a 0.22 $\mu$ mpore-size filter, and the resulting filtrate was considered as biofilm supernatants. A culture was also established where the $\mathrm{pH}$ of the medium supplemented with glucose was adjusted to 7.5. For genomic DNA (gDNA) solution, overnight cultures of Newman were centrifuged at $12,000 \mathrm{~g}$ for $5 \mathrm{~min}$ and the resulting precipitate was washed twice with sterile normal saline, resuspended in TE buffer, and boiled at $100^{\circ} \mathrm{C}$ for $10 \mathrm{~min}$. The suspension was centrifuged at $12,000 \mathrm{~g}$ for $15 \mathrm{~min}$ and the supernatant was considered as gDNA solution. Twenty microliters of gDNA solution was added to $180 \mu \mathrm{L}$ biofilm supernatant and incubated at $37^{\circ} \mathrm{C}$ for $30 \mathrm{~min}$. The remaining gDNA in the solution was quantified by real-time PCR as described above.

\section{Survival of S. aureus Newman Under Acidic Conditions}

Staphylococcus aureus Newman was grown in LB medium overnight, then $3 \mathrm{~mL}$ of this culture was incubated in 15$\mathrm{mL}$ tubes with $25 \mathrm{mM}$ glucose, $20 \mathrm{mM}$ lactic acid (Sigma), or $20 \mathrm{mM}$ acetic acid (Sigma) for $48 \mathrm{~h}$. CFUs of the cultures were determined by performing serial dilutions and plating $5 \mu \mathrm{L}$ on BA or L-form induced agar plates (LIA, Caring Trading Co., Ltd., 

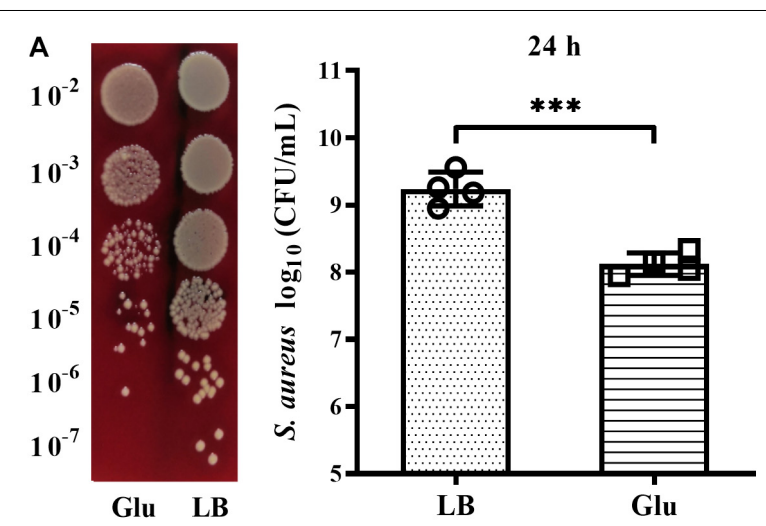

C

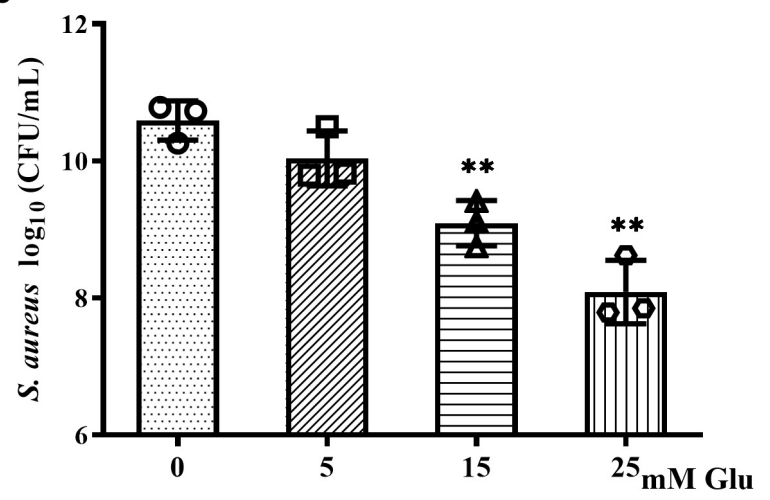

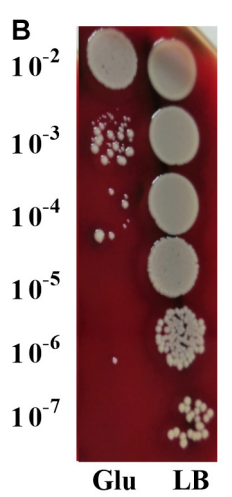

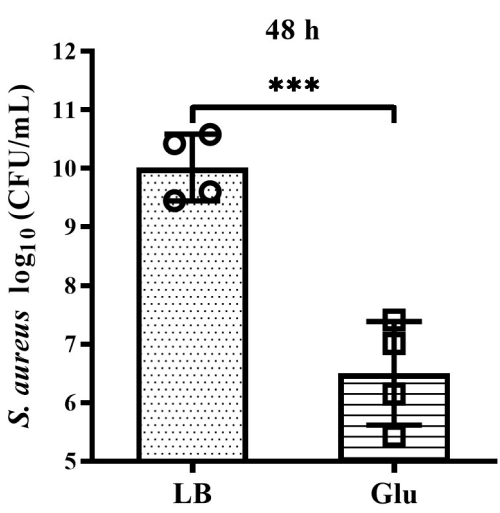

D

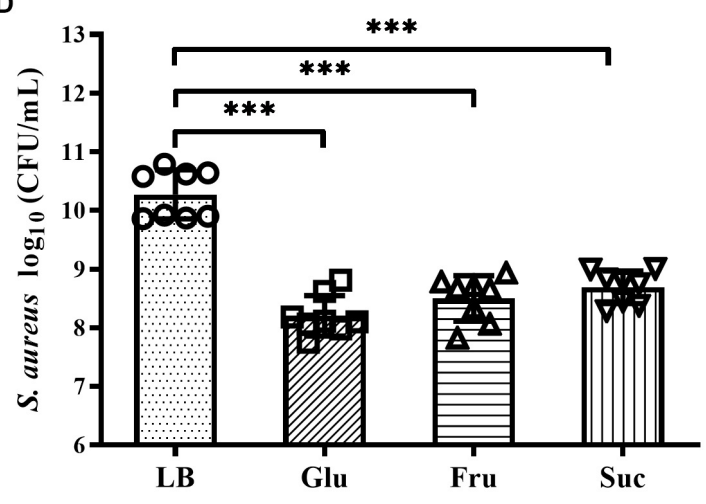

FIGURE 2 | Glucose reduced growth and survival of $S$. aureus Newman. (A) CFUs of S. aureus Newman cultured in LB medium with or without 25 mM glucose for $24 \mathrm{~h}$, with serial dilution and plating on BA. (B) CFUs of $S$. aureus Newman cultured in LB medium with or without $25 \mathrm{mM}$ glucose for $48 \mathrm{~h}$. (C) CFUs of $\mathrm{S}$. aureus Newman cultured in LB medium supplemented with 0, 5, 15, or $25 \mathrm{mM}$ glucose for $24 \mathrm{~h}$. (D) CFUs of eight clinical $S$. aureus isolates from diabetic patients with foot ulcers cultured in LB medium with or without $25 \mathrm{mM}$ glucose, $25 \mathrm{mM}$ fructose, or $12.5 \mathrm{mM}$ sucrose for $24 \mathrm{~h}$. ** $p<0.01$; *** $p<0.001$.

Guangdong, China). Plates were incubated overnight at $37^{\circ} \mathrm{C}$ before enumeration of colonies.

\section{Gram-Staining Analysis}

A smear of bacterial culture $(50 \mu \mathrm{L})$ was placed on a glass slide, thoroughly air dried, heat fixed, and then stained with a commercial Gram-staining solution (BASO Diagnostics, China). Briefly, the staining process comprised $10 \mathrm{~s}$ in crystal violet solution, $10 \mathrm{~s}$ in iodine solution, $20 \mathrm{~s}$ wash in decolorizer, then a final counter stain with safranin solution for $10 \mathrm{~s}$. The stained slides were examined by an microscope $(1000 \times)$.

\section{Transmission Electron Microscopy}

Bacteria were collected by centrifugation at $3000 \mathrm{~g}$ for $10 \mathrm{~min}$ and were washed twice with phosphate-buffered saline (PBS). These bacteria were resuspended in $2.5 \%$ glutaraldehyde solution with Millonig's phosphate buffer and shipped to the Transmission Electron Microscopy (TEM) Laboratory at the Pathology Department of Xiangya Hospital, Changsha, Hunan. Specimens were examined and photographed on a Hitachi HT7700 electron microscope.

\section{Lysostaphin and Lysozyme Lysis Assay}

Lysostaphin lysis assays were performed as previously reported with minor modifications (Grundling et al., 2006). Briefly, overnight cultures of S. aureus, S. epidermidis, or S. haemolyticus were diluted 1:100 into fresh LB medium with or without $25 \mathrm{mM}$ glucose and were cultured at $37^{\circ} \mathrm{C}, 180 \mathrm{rpm}$ for $24 \mathrm{~h}$. For inhibition of glycolysis by 2-deoxy-D-glucose (2-DG), overnight cultures of $S$. aureus were diluted 1:100 into fresh LB medium containing $15 \mathrm{mM}$ glucose and $60 \mathrm{mM}$ 2-DG (Sigma) and were cultured at $37^{\circ} \mathrm{C}, 180 \mathrm{rpm}$ for $8 \mathrm{~h}$. These above cultures were then washed twice with PBS and resuspended to an $\mathrm{OD}_{630}$ of 1.82.1. This value was set as $100 \%$ at $0 \mathrm{~min}$. Lysostaphin (Sigma) or lysozyme (Sigma) was added at final concentrations of 10 and $50 \mu \mathrm{g} / \mathrm{mL}$, respectively. $\mathrm{OD}_{630}$ measurements were recorded at timed intervals, and data were plotted as percent $\mathrm{OD}_{630}$ values of the initial reading.

\section{Real-Time RT-PCR}

Total RNA extraction was extracted from S. aureus Newman as described previously (Beltrame et al., 2015). Initially, cells were lysed using lysostaphin and Trizol (Sigma), then total RNA was 
extracted and purified using a nucleic acid extraction kit (Enzo Life Sciences) according to the manufacturer's instructions. The concentration of the extracted RNA was evaluated by using a NanoDrop 1000 (Thermo Fisher Scientific). A total of $1 \mu \mathrm{g}$ RNA was then reverse transcribed to cDNA using a reverse transcription kit (TransGen Biotech, Beijing, China) according to the manufacturer's instructions. Briefly, real-time PCR was conducted under the following conditions: pre-denaturation at $94^{\circ} \mathrm{C}$ for $5 \mathrm{~min} ; 40$ cycles of denaturation at $94^{\circ} \mathrm{C}$ for $30 \mathrm{~s}$, annealing at $57^{\circ} \mathrm{C}$ for $20 \mathrm{~s}$, and extension at $72^{\circ} \mathrm{C}$ for $10 \mathrm{~s}$. Relative expression was determined by using the $2^{-\Delta \Delta \mathrm{Ct}}$ method. The level of transcription was determined relative to the expression of $g y r B$. Primers used for this real-time PCR were reported in a previous study (Supplementary Table 1; Brahma et al., 2019).

\section{Statistical Analysis}

All data produced in this study was normal distributions, and an unpaired Student's $t$-test was used to further evaluate the difference between two groups. All probabilities were two-tailed and $p<0.05$ was considered as significant. Data were presented as the mean \pm standard deviation (SD). Statistical analyses were performed by using GraphPad Prism version 5 (GraphPad Software, San Diego, CA).

\section{RESULTS}

\section{Reduced eDNA Release of S. aureus by Glucose}

Addition of glucose to the growth medium simulates biofilm formation by $S$. aureus (Boles and Horswill, 2008). However, in this study, the CFUs of biofilms of $S$. aureus Newman were unaffected by addition of $15 \mathrm{mM}$ glucose (Figure 1A). eDNA is an important component of biofilms, and which was markedly decreased by glucose (Figures 1B,C), concordant with results from a previous study (Sugimoto et al., 2018). Within biofilms, this reduction of eDNA by glucose was dose-dependent (Figure 1D). eDNA levels within biofilms are determined by eDNA release and degradation. Therefore, nuclease activity in the biofilm supernatants of $S$. aureus Newman was examined and was found to decrease significantly in the presence of glucose compared with LB medium (Figure 1E). Amount of acidic metabolites produced during the biofilm formation under high glucose condition may affect the nuclease activity of biofilm supernatants. After adjusting the $\mathrm{pH}$ of biofilm supernatants with glucose to 7.5 , the nuclease activity was restored to almost levels observed in LB medium (Figure 1E). These data indirectly indicate that glucose reduces eDNA release but not eDNA degradation.

\section{Reduced Growth and Survival of S. aureus Under High Glucose Conditions}

The growth and survival of planktonic S. aureus Newman in the presence of glucose was examined. After culture for $24 \mathrm{~h}$, CFUs of $S$. aureus Newman were reduced nearly 10 -fold following addition of $25 \mathrm{mM}$ glucose (Figure 2A). An increase in culture time to $48 \mathrm{~h}$ did not affect the CFUs of $S$. aureus Newman from the medium without glucose, but the CFUs of S. aureus Newman from the medium with glucose were further reduced by more than 1000-fold at this time point (Figures 2A,B). These data indicated that growth and survival of $S$. aureus Newman was significantly decreased by high concentrations of glucose. Next, the growth of $S$. aureus Newman in the presence of different concentrations of glucose was investigated. Reduced growth of this strain in the presence of glucose was observed to be dosedependent (Figure 2C). Finally, the growth of eight clinical

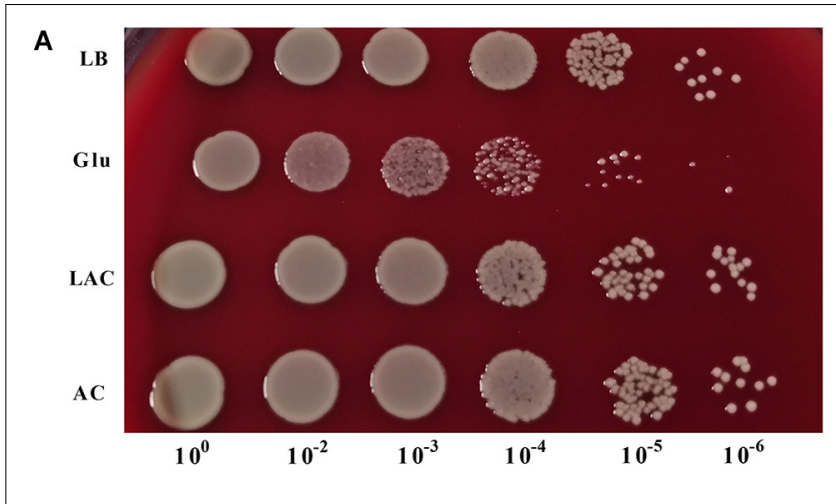

B

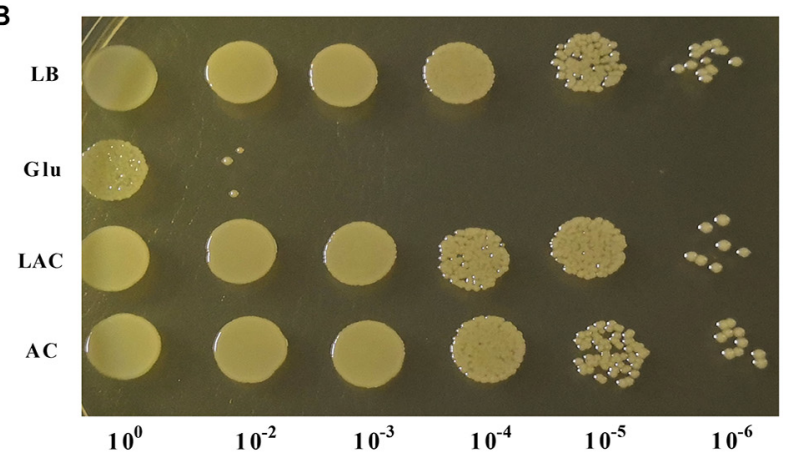

C

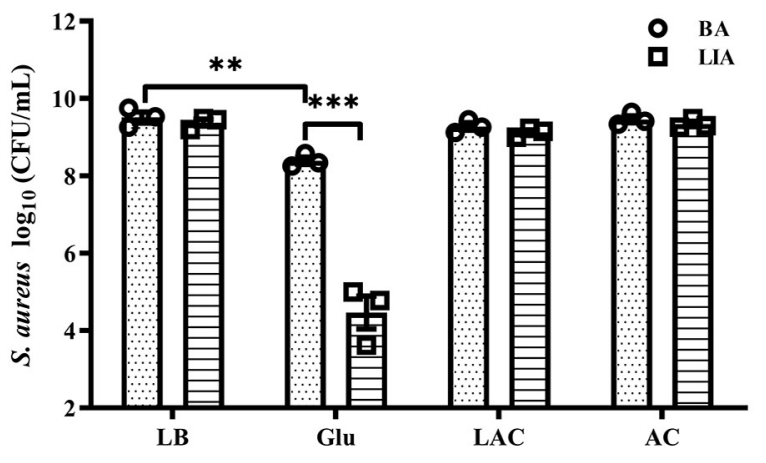

FIGURE 3 | Survival of $S$. aureus Newman was unaffected by organic acid. S. aureus Newman was grown overnight in LB medium, then $25 \mathrm{mM}$ glucose, $20 \mathrm{mM}$ lactic acid, or $20 \mathrm{mM}$ acetic acid was added to the medium and the cultures were incubated for a further $48 \mathrm{~h}$. CFUs were then determined by serial dilution and plating on BA (A,C) and LIA plates (B,C). ${ }^{* *} p<0.01$; $* * * p<0.001$. 
A

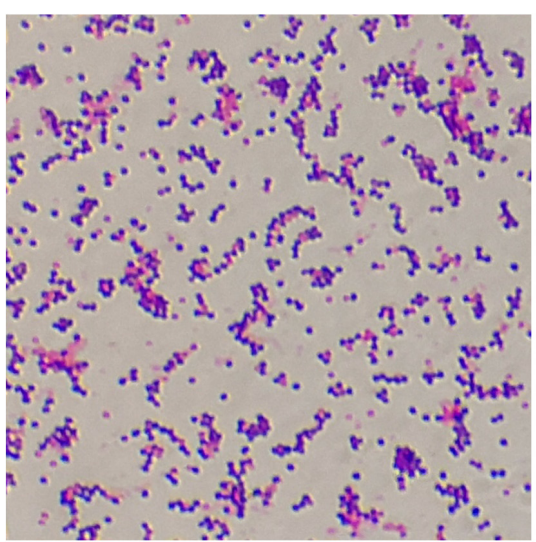

LB

C

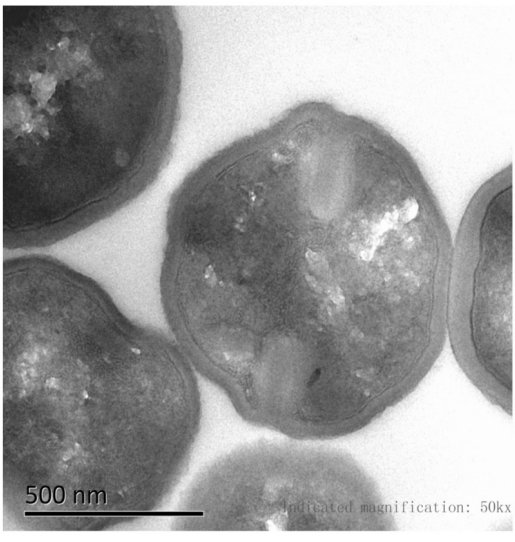

LB

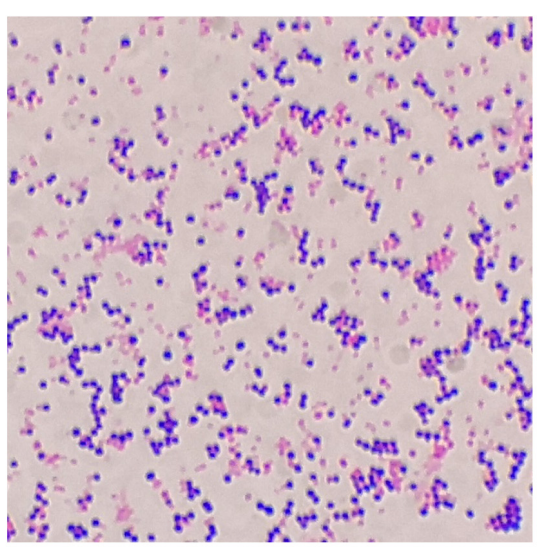

Glu

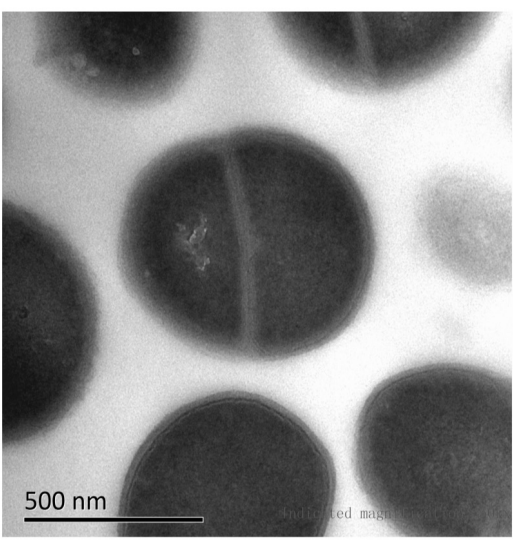

Glu

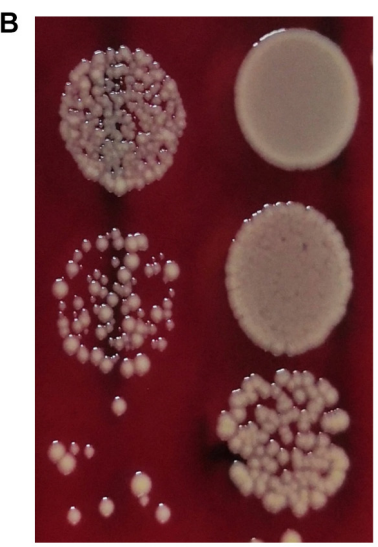

Glu

LB

FIGURE 4 | Glucose reduced the thickness of the cell wall of S. aureus Newman. S. aureus Newman was cultured in LB medium with or without 25 mM glucose. (A) After cultured for 48 h, Gram-staining was used to examine morphology changes of $S$. aureus by glucose. (B) After 24 h, culture medium were diluted and plated on BA. (C) After $48 \mathrm{~h}$, bacterial cells were analyzed by TEM, and the thickness of the cell wall was measured. Graphs show representative results from two independent experiments. ${ }^{* * *} p<0.001$.

S. aureus isolates from patients with diabetic foot ulcers was examined in the presence and absence of glucose, and a high concentration of glucose $(25 \mathrm{mM})$ was found to significantly reduce growth of these clinical isolates after culture for $24 \mathrm{~h}$ (Figure 2D). Similar results were observed following addition of fructose or sucrose (Figure 2D). These data indicate that the growth and survival of $S$. aureus is impaired under high glucose conditions.

\section{Survival of S. aureus Under Acidic Conditions}

The $\mathrm{pH}$ of growth medium of $S$. aureus Newman in the planktonic state dropped to 5.0 after culture for $8 \mathrm{~h}$ in the presence of $25 \mathrm{mM}$ glucose (data not shown). To determine whether acidic conditions affected survival of $S$. aureus, $20 \mathrm{mM}$ exogenous lactic acid or acetic acid was added to overnight cultures of $S$. aureus Newman, resulting in the culture medium reaching a $\mathrm{pH}$ of 5.0 (data not shown). After culture for $48 \mathrm{~h}$ under these acidic conditions, the bacteria were serially diluted and plated on BA and LIA plates. On the BA plates, CFUs of $S$. aureus Newman significantly decreased following addition of $25 \mathrm{mM}$ glucose, but were unchanged following addition of exogenous lactic acid or acetic acid (Figures 3A,C). Similar results were observed on LIA plates (Figures 3B,C). These data indicate that survival of $S$. aureus Newman under high glucose conditions is unaffected by acidic metabolites.

\section{Reduced Cell-Wall Thickness of S. aureus Under High Glucose Conditions}

Gram-positive bacteria are known to have a thick cell wall, whereas Gram-negative bacteria have a thin cell wall. Changes in the morphology of $S$. aureus in the presence of glucose were firstly investigated using Gram-staining. S. aureus is Gram-positive, but the proportion of Gram-stain-negative bacteria from the culture medium with glucose was markedly increased compared to those 


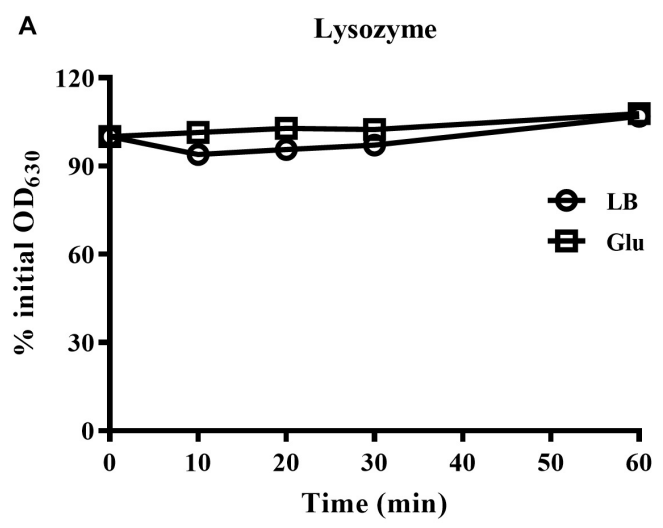

C

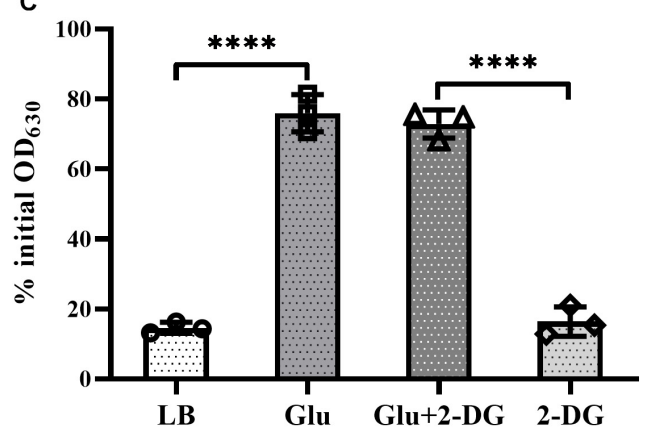

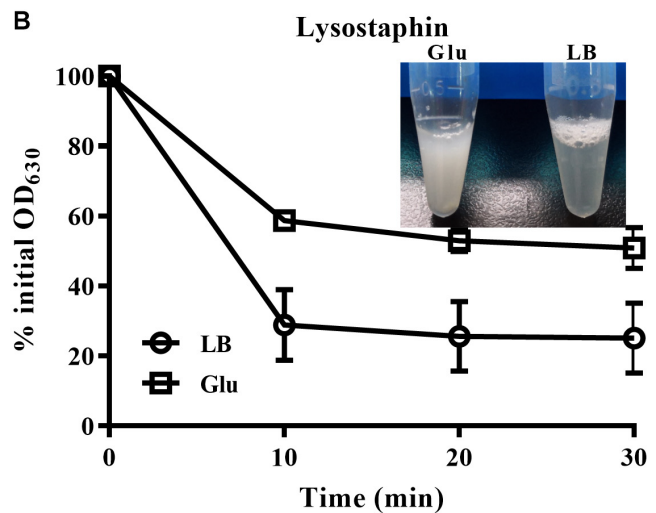

D

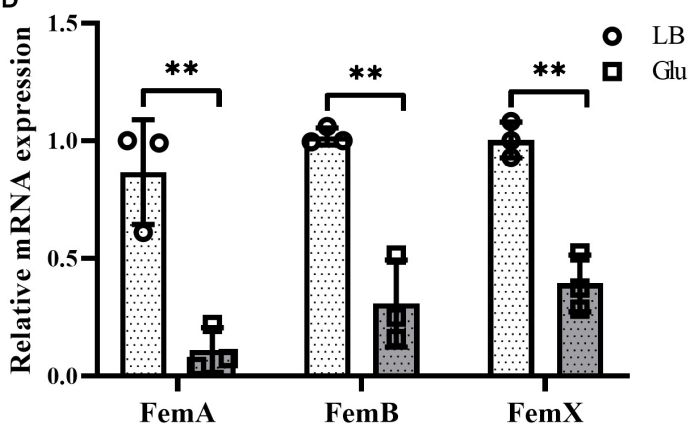

FIGURE 5 | Glucose reduced expression of pentaglycine. S. aureus Newman was cultured in LB medium with or without 15 mM glucose for 24 h. Bacterial cells were collected by centrifugation and resuspended in PBS buffer, with lysozyme (A) or lysostaphin (B) added at a final concentration of 50 and $10 \mu \mathrm{g} / \mathrm{mL}$, respectively. $\mathrm{OD}_{630}$ measurements were taken at timed intervals. (C) Lysostaphin resistance of $\mathrm{S}$. aureus Newman in medium supplemented with 15 mM glucose was unaffected by the addition of $60 \mathrm{mM}$ 2-DG. (D) $\mathrm{S}$. aureus Newman was cultured in LB medium with or without $15 \mathrm{mM}$ glucose for 6 h, and bacterial cells were collected for analysis of mRNA expression of femA, femB, and femX. ${ }^{* *} p<0.01$; ${ }^{* * *} p<0.0001$.

from LB medium (Figure 4A). To exclude the possibility of Gram-negative bacteria as contaminants, the culture medium were diluted, plated on BA, and incubated overnight. Only different-sized colonies of $S$. aureus grew on BA after overnight culture (Figure 4B). TEM analysis was used to further determine structural changes in the cell wall of $S$. aureus Newman in the presence of glucose. As shown in Figure 4C, the thickness of the bacterial cell wall was significantly decreased by a high concentration of glucose. These data indicate that $S$. aureus displays defects in the cell wall under high glucose conditions.

\section{Reduced Pentaglycine Expression of S. aureus Under High Glucose Conditions}

Staphylococcus aureus is completely resistant to the hydrolytic activity of lysozyme (Bera et al., 2007). This resistance of $S$. aureus to lysozyme was unaffected by the addition of glucose (Figure 5A). In contrast, $S$. aureus is susceptible to the enzyme lysostaphin, which recognizes the pentaglycine bridges between peptidoglycan chains, and then drives the preferential digestion of cell walls (Grundling and Schneewind, 2006). However, cells of $S$. aureus from the culture medium with glucose were highly resistant to lysostaphin compared to those from LB medium (Figure 5B). In addition, lysostaphin resistance induced by glucose was unaffected by the inhibitor of glycolysis, 2DG (Figure 5C). These data suggest that the expression of pentaglycine bridges in $S$. aureus may be affected by glucose. Members of the factor essential for methicillin resistance $A B X$ $(f e m A B X)$ family are required for synthesis of pentaglycine crossbridges in staphylococci (Monteiro et al., 2019). Therefore, expression of $f e m A, f e m B$, and femX mRNA was examined in the cultures of $S$. aureus. As shown in Figure 5D, mRNA levels of $f e m A, f e m B$, and $f e m X$ were significantly decreased in the presence of glucose. These data indicate that high glucose reduces the synthesis of pentaglycine bridges between peptidoglycan chains in $S$. aureus.

\section{The Unique Cell-Wall Structure Is Associated With the Reduced Growth of Staphylococci by Glucose}

Peptidoglycan is the main component of bacterial cell walls, the composition of which varies considerably between species. Staphylococci possess highly cross-linked peptidoglycan due to the presence of pentaglycine bridges. To investigate whether 


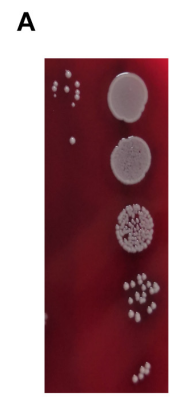

Glu LB

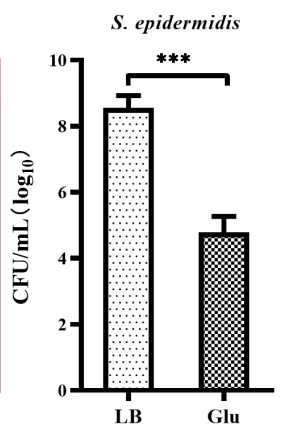

E. faecalis

D

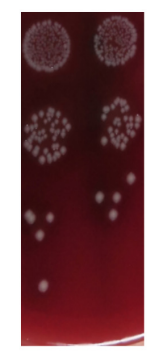

Glu LB

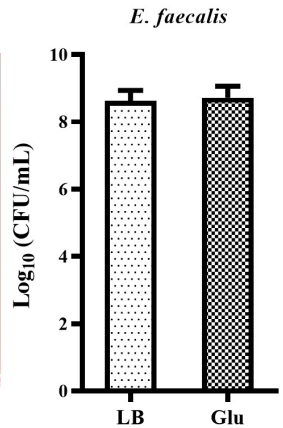

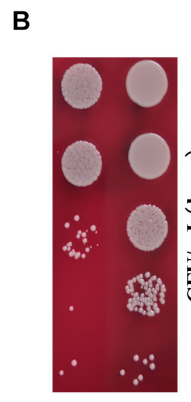

Glu LB

E

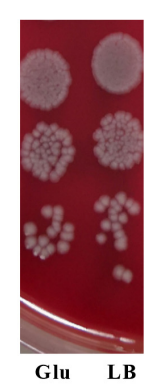

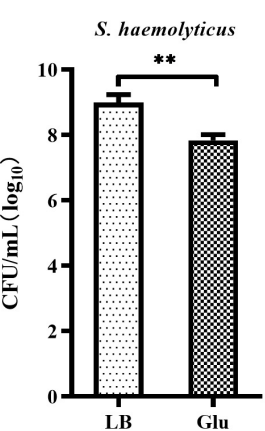

C

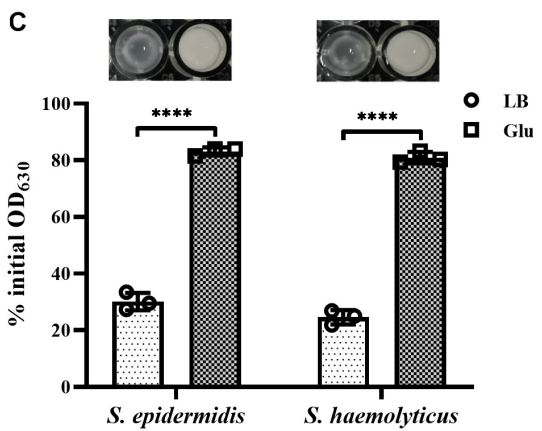

F
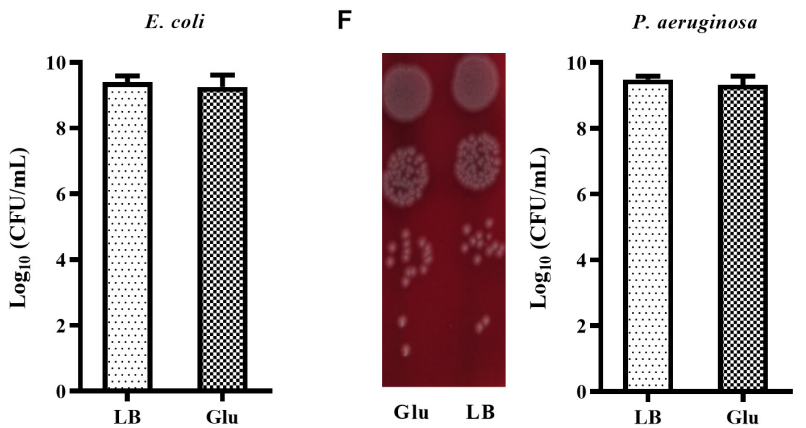

FIGURE 6 | Glucose reduced the growth of staphylococci. (A) S. epidermidis RP62A and (B) S. haemolyticus SH01 were cultured in LB medium with or without $25 \mathrm{mM}$ glucose for $48 \mathrm{~h}$, then the CFUs of the cultures were determined by serial dilution and plating on BA. (C) S. epidermidis RP62A and S. haemolyticus SH01 were cultured in LB medium with or without $25 \mathrm{mM}$ glucose for $24 \mathrm{~h}$. The bacterial cells were collected by centrifugation, resuspended in PBS buffer, and lysostaphin was added at a final concentration of $10 \mu \mathrm{g} / \mathrm{mL}$. OD 630 measurements were recorded after 30 min. (D) Enterococcus faecalis ATCC29212, (E) Escherichia coli ATCC25922, and (F) P. aeruginosa ATCC27853 were cultured in LB medium with or without 25 mM glucose for 48 h, then CFUs of the cultures were determined by serial dilution and plating on BA. ${ }^{* *} p<0.01 ;{ }^{* *} p<0.001 ;{ }^{* * *} p<0.0001$.

glucose affected the growth of other staphylococci. In addition to $S$. aureus, the growth of S. epidermidis and S. haemolyticus in the presence of $25 \mathrm{mM}$ glucose was examined. Addition of glucose significantly reduced the growth of $S$. epidermidis and S. haemolyticus (Figures 6A,B). Furthermore, S. epidermidis and S. haemolyticus were highly resistant to lysostaphin in the LB medium with $25 \mathrm{mM}$ glucose (Figure 6C). However, growth of E. faecalis and Gram-negative bacteria, such as E. coli and $P$. aeruginosa, was unaffected by glucose (Figures 6D-F). These data indicate that the unique cell-wall structure of staphylococci results in their reduced growth in the presence of glucose.

\section{DISCUSSION}

High-glucose-induced cytotoxicity has been observed in various eukaryotic cells, such as endothelial cells and fibroblasts (Gao et al., 2015; Sedlic et al., 2017; Buranasin et al., 2018). In this study, a high concentration of glucose was found to significantly reduce growth and survival of planktonic $S$. aureus. A previous study reported that growth of $E$. coli in unbuffered LB medium was markedly decreased by the addition of $11.11 \mathrm{mM}$ glucose (Kwiatkowska et al., 2008). The $\mathrm{pH}$ of the growth medium dropped to 5.0 in the presence of $25 \mathrm{mM}$ glucose in this study and our previous study (Luo et al., 2019). These data suggested that acidic metabolites might contribute to the reduced growth and survival of $S$. aureus by glucose. The lactic acid content of the culture medium during aggregation of $S$. aureus induced by glucose was less than $15 \mathrm{mM}$ (Luo et al., 2019), therefore $20 \mathrm{mM}$ lactic acid or acetic acid was added into the overnight cultures of $S$. aureus, resulting in the $\mathrm{pH}$ of the cultures decreasing to 5.0. Survival of $S$. aureus during stationary phase was unaffected by these acidic conditions, indicating that the reduced growth and survival of $S$. aureus under high glucose conditions is not due to the presence of acidic metabolites. Contrary to these data, murine footpad infection models revealed that hyperglycemia favors bacterial survival (Tuchscherr et al., 2018). Therefore, other internal and external factors, such as stain lineage and serum components, also affect the growth and survival of strains of S. aureus (Croes et al., 2009; Yin et al., 2017).

Staphylococcus aureus forms robust biofilms in growth medium supplemented with glucose, and the increased biofilm formation induced by glucose has a threshold response at clinically important concentrations (Waldrop et al., 2014). During biofilm formation, a population of cells release extracellular matrix, such as extracellular proteins and eDNA, to form multicellular clusters. The eDNA component within biofilms is released into the supernatant when the biofilms are 
suspended in a buffer with a higher pH (Dengler et al., 2015); this enables eDNA within biofilms to be experimentally separated from gDNA. Herein, glucose $(>3 \mathrm{mM})$ significantly reduced eDNA levels within biofilms, and the amount of glucose was less than that reported in a previous study (Sugimoto et al., 2018). A previous study demonstrated that $22.22 \mathrm{mM}$ glucose could repress nuclease transcription and prevent nuclease accumulation (Kiedrowski et al., 2011). In our study, nuclease activity of biofilm supernatants was significantly reduced in the presence of $15 \mathrm{mM}$ glucose compared with LB medium, and this activity could be restored almost to levels recorded in LB medium by adjusting the $\mathrm{pH}$ of the culture solution to 7.5. Therefore, eDNA does not contribute to the increase in biofilm formation induced by glucose; other factors are involved in this process.

In addition to $S$. aureus, growth of strains of S. epidermidis and $S$. haemolyticus was also markedly decreased by glucose, while growth of E. faecalis, E. coli, and P. aeruginosa was unaffected by glucose. Furthermore, like S. aureus, strains of S. epidermidis and S. haemolyticus were highly resistant to lysostaphin under high glucose conditions. Pentaglycine bridges, which are cleaved by lysostaphin, occur in the cell envelope of staphylococci but not in other bacterial species. Therefore, the unique cell-wall structure of staphylococci contributes to the reduced growth and survival of $S$. aureus in high glucose conditions.

The thickness of the cell wall of $S$. aureus was significantly reduced under high glucose conditions. Staphylococci possess highly cross-linked peptidoglycan due to the presence of the bridges containing five glycine molecules; these pentaglycine bridges are synthesized by the fem $A B X$ family (Rohrer et al., 1999; Monteiro et al., 2019). Deletion of $f e m A B$ genes reduces crosslinking between peptidoglycan chains, and results in bacterial strain being highly lysostaphin resistant (Stranden et al., 1997). Cells from the medium supplemented with glucose were highly resistant to lysostaphin. In addition, mRNA expression of genes of the fem $A B X$ family in $S$. aureus Newman was significantly reduced by glucose compared with expression levels in LB medium. Therefore, culture of $S$. aureus under high glucose conditions leads to decreased pentaglycine expression in the bacteria and subsequent lysostaphin resistance.

Lysostaphin-resistant strains of $S$. aureus exhibit a reduced rate of exponential growth compared to lysostaphin-sensitive strains (Kusuma et al., 2007). Furthermore, cells of S. aureus do not survive without pentaglycine bridges, appearing as pseudomulticellular forms that eventually lyse due to extensive membrane rupturing (Monteiro et al., 2019). Our previous study reported that under high glucose conditions, cells of $S$. aureus formed multicellular arrangements and sank to the bottom of the culture vessel (Luo et al., 2019). Therefore, the reduced growth and survival of strains of $S$. aureus in high glucose is due to decreased expression of pentaglycine.

\section{REFERENCES}

Bayer, A. S., Schneider, T., and Sahl, H. G. (2013). Mechanisms of daptomycin resistance in Staphylococcus aureus: role of the cell membrane and cell wall. Ann. N. Y. Acad. Sci. 1277, 139-158. doi: 10.1111/j.1749-6632.2012.06819.x
Lysostaphin shows rapid bactericidal activity against methicillin-susceptible and -resistant strains of $S$. aureus, with minimum inhibitory concentrations (MICs) ranging from 0.03 to $2 \mu \mathrm{g} / \mathrm{mL}$, as determined using an agar dilution assay (Yang et al., 2007). Recent studies have reported that lysostaphin combined with other agents, such as LL-37 peptide, liposomal vancomycin, and nisin, are effective against $S$. aureus infection (Ceotto-Vigoder et al., 2016; Hajiahmadi et al., 2019; Sadeghi et al., 2019). However, the MIC values of lysostaphin are greatly increased by serial subculture (Gutierrez et al., 2020). Moreover, high levels of glucose and rich medium also lead to lysostaphin resistance (Wu et al., 2019). Therefore, utilization of lysostaphin to treat $S$. aureus infections should consider the factors affecting lysostaphin sensitivity.

\section{DATA AVAILABILITY STATEMENT}

The raw data supporting the conclusions of this article will be made available by the authors, without undue reservation, to any qualified researcher.

\section{AUTHOR CONTRIBUTIONS}

ZL, YuW, and YoW designed the study and analyzed the results. ZL, SY, TC, and PS conducted the experiments. ZL and YuW wrote the manuscript. SY, TC, PS, and YoW reviewed the manuscript. All authors contributed to the article and approved the submitted version.

\section{FUNDING}

This study was supported by the New Xiangya Talent Project of the Third Xiangya Hospital of Central South University (grant no. JY201713), the Natural Science Foundation of Hunan Province (grant no. 2019JJ50925), and the National Natural Science Foundation of China (grant no. 81501375). The funding bodies had no role in the design of the study, nor in the collection, analysis, and interpretation of data, nor in the writing of the manuscript.

\section{SUPPLEMENTARY MATERIAL}

The Supplementary Material for this article can be found online at: https://www.frontiersin.org/articles/10.3389/fmicb. 2020.537290/full\#supplementary-material

Beltrame, C. O., Cortes, M. F., Bandeira, P. T., and Figueiredo, A. M. (2015). Optimization of the RNeasy Mini Kit to obtain high-quality total RNA from sessile cells of Staphylococcus aureus. Braz. J. Med. Biol. Res. 48, 1071-1076. doi: 10.1590/1414-431X2015 4734 
Bera, A., Biswas, R., Herbert, S., Kulauzovic, E., Weidenmaier, C., Peschel, A., et al. (2007). Influence of wall teichoic acid on lysozyme resistance in Staphylococcus aureus. J. Bacteriol. 189, 280-283. doi: 10.1128/JB.01221-06

Bertsche, U., Weidenmaier, C., Kuehner, D., Yang, S. J., Baur, S., Wanner, S., et al. (2011). Correlation of daptomycin resistance in a clinical Staphylococcus aureus strain with increased cell wall teichoic acid production and D-alanylation. Antimicrob. Agents Chemother. 55, 3922-3928. doi: 10.1128/AAC.01226-10

Boles, B. R., and Horswill, A. R. (2008). Agr-mediated dispersal of Staphylococcus aureus biofilms. PLoS Pathog. 4:e1000052. doi: 10.1371/journal.ppat.1000052

Brahma, U., Sharma, P., Murthy, S., Sharma, S., Chakraborty, S., Appalaraju, S. N., et al. (2019). Decreased expression of femXAB genes and fnbp mediated biofilm pathways in OS-MRSA clinical isolates. Sci. Rep. 9:16028. doi: 10.1038/s41598019-52557-z

Buranasin, P., Mizutani, K., Iwasaki, K., Na Mahasarakham, C. P., Kido, D., Takeda K., et al. (2018). High glucose-induced oxidative stress impairs proliferation and migration of human gingival fibroblasts. PLoS One 13:e0201855. doi: 10.1371/ journal.pone.0201855

Ceotto-Vigoder, H., Marques, S. L., Santos, I. N., Alves, M. D., Barrias, E. S., Potter, A., et al. (2016). Nisin and lysostaphin activity against preformed biofilm of Staphylococcus aureus involved in bovine mastitis. J. Appl. Microbiol. 121, 101-114. doi: 10.1111/jam.13136

Chen, C., Fan, H., Huang, Y., Peng, F., Fan, H., Yuan, S., et al. (2014). Recombinant lysostaphin protects mice from methicillin-resistant Staphylococcus aureus pneumonia. Biomed. Res. Int. 2014:602185. doi: 10.1155/2014/602185

Croes, S., Deurenberg, R. H., Boumans, M. L., Beisser, P. S., Neef, C., and Stobberingh, E. E. (2009). Staphylococcus aureus biofilm formation at the physiologic glucose concentration depends on the $S$. aureus lineage. BMC Microbiol. 9:229. doi: 10.1186/1471-2180-9-229

Dengler, V., Foulston, L., DeFrancesco, A. S., and Losick, R. (2015). An Electrostatic Net Model for the Role of Extracellular DNA in Biofilm Formation by Staphylococcus aureus. J. Bacteriol. 197, 3779-3787. doi: 10.1128/JB.00726-15

Francius, G., Domenech, O., Mingeot-Leclercq, M. P., and Dufrene, Y. F. (2008) Direct observation of Staphylococcus aureus cell wall digestion by lysostaphin. J. Bacteriol. 190, 7904-7909. doi: 10.1128/JB.01116-08

Gao, Y., Zhang, J., Li, G., Xu, H., Yi, Y., Wu, Q., et al. (2015). Protection of vascular endothelial cells from high glucose-induced cytotoxicity by emodin. Biochem. Pharmacol. 94, 39-45. doi: 10.1016/j.bcp.2015.01.006

Grundling, A., Missiakas, D. M., and Schneewind, O. (2006). Staphylococcus aureus mutants with increased lysostaphin resistance. J. Bacteriol. 188, 6286-6297. doi: 10.1128/JB.00457-06

Grundling, A., and Schneewind, O. (2006). Cross-linked peptidoglycan mediates lysostaphin binding to the cell wall envelope of Staphylococcus aureus. J. Bacteriol. 188, 2463-2472. doi: 10.1128/JB.188.7.2463-2472.2006

Gutierrez, D., Garrido, V., Fernandez, L., Portilla, S., Rodriguez, A., Grillo, M. J., et al. (2020). Phage Lytic Protein LysRODI Prevents Staphylococcal Mastitis in Mice. Front. Microbiol. 11:7. doi: 10.3389/fmicb.2020.00007

Hajiahmadi, F., Alikhani, M. Y., Shariatifar, H., Arabestani, M. R., and Ahmadvand, D. (2019). The bactericidal effect of lysostaphin coupled with liposomal vancomycin as a dual combating system applied directly on methicillinresistant Staphylococcus aureus infected skin wounds in mice. Int. J. Nanomed. 14, 5943-5955. doi: 10.2147/IJN.S214521

Kiedrowski, M. R., Kavanaugh, J. S., Malone, C. L., Mootz, J. M., Voyich, J. M., Smeltzer, M. S., et al. (2011). Nuclease modulates biofilm formation in community-associated methicillin-resistant Staphylococcus aureus. PLoS One 6:e26714. doi: 10.1371/journal.pone.0026714

Kusuma, C., Jadanova, A., Chanturiya, T., and Kokai-Kun, J. F. (2007). Lysostaphin-resistant variants of Staphylococcus aureus demonstrate reduced fitness in vitro and in vivo. Antimicrob. Agents Chemother. 51, 475-482. doi: 10.1128/AAC.00786-06

Kwiatkowska, J., Matuszewska, E., Kuczynska-Wisnik, D., and Laskowska, E. (2008). Aggregation of Escherichia coli proteins during stationary phase depends on glucose and oxygen availability. Res. Microbiol. 159, 651-657. doi: 10.1016/j.resmic.2008.09.008

Labischinski, H. (1992). Consequences of the interaction of beta-lactam antibiotics with penicillin binding proteins from sensitive and resistant Staphylococcus aureus strains. Med. Microbiol. Immunol. 181, 241-265. doi: 10.1007/ bf00198846
Liu, Y., Huang, H., Gao, R., and Liu, Y. (2020). Dynamic Phenotypes and Molecular Mechanisms to Understand the Pathogenesis of Diabetic Nephropathy in Two Widely Used Animal Models of Type 2 Diabetes Mellitus. Front. Cell Dev. Biol. 8:172. doi: $10.3389 /$ fcell.2020.00172

Luo, Z., Chen, M., Chen, T., She, P., and Wu, Y. (2019). Lactic Acid Produced by Glycolysis Contributed to Staphylococcus aureus Aggregation Induced by Glucose. Curr. Microbiol. 76, 607-612. doi: 10.1007/s00284-019-01666-Z

Merino, N., Toledo-Arana, A., Vergara-Irigaray, M., Valle, J., Solano, C., Calvo, E., et al. (2009). Protein A-mediated multicellular behavior in Staphylococcus aureus. J. Bacteriol. 191, 832-843. doi: 10.1128/JB.01222-08

Monteiro, J. M., Covas, G., Rausch, D., Filipe, S. R., Schneider, T., Sahl, H. G., et al. (2019). The pentaglycine bridges of Staphylococcus aureus peptidoglycan are essential for cell integrity. Sci. Rep. 9:5010. doi: 10.1038/s41598-019-41461-1

Oluola, O., Kong, L., Fein, M., and Weisman, L. E. (2007). Lysostaphin in treatment of neonatal Staphylococcus aureus infection. Antimicrob. Agents Chemother. 51, 2198-2200. doi: 10.1128/AAC.00506-06

Prax, M., Mechler, L., Weidenmaier, C., and Bertram, R. (2016). Glucose Augments Killing Efficiency of Daptomycin Challenged Staphylococcus aureus Persisters. PLoS One 11:e0150907. doi: 10.1371/journal.pone.0150907

Richardson, A. R., Libby, S. J., and Fang, F. C. (2008). A nitric oxide-inducible lactate dehydrogenase enables Staphylococcus aureus to resist innate immunity. Science 319, 1672-1676. doi: 10.1126/science.1155207

Rohrer, S., Ehlert, K., Tschierske, M., Labischinski, H., and Berger-Bachi, B. (1999). The essential Staphylococcus aureus gene fmhB is involved in the first step of peptidoglycan pentaglycine interpeptide formation. Proc. Natl. Acad. Sci. U S A. 96, 9351-9356. doi: 10.1073/pnas.96.16.9351

Sadeghi, S., Bakhshandeh, H., Ahangari Cohan, R., Peirovi, A., Ehsani, P., and Norouzian, D. (2019). Synergistic Anti-Staphylococcal Activity Of Niosomal Recombinant Lysostaphin-LL-37. Int. J. Nanomed. 14, 9777-9792. doi: 10.2147/ IJN.S230269

Sedlic, F., Muravyeva, M. Y., Sepac, A., Sedlic, M., Williams, A. M., Yang, M., et al. (2017). Targeted Modification of Mitochondrial ROS Production Converts High Glucose-Induced Cytotoxicity to Cytoprotection: Effects on Anesthetic Preconditioning. J. Cell Physiol. 232, 216-224. doi: 10.1002/jcp.25413

Stranden, A. M., Ehlert, K., Labischinski, H., and Berger-Bachi, B. (1997). Cell wall monoglycine cross-bridges and methicillin hypersusceptibility in a femAB null mutant of methicillin-resistant Staphylococcus aureus. J. Bacteriol. 179, 9-16. doi: 10.1128/jb.179.1.9-16.1997

Sugimoto, S., Sato, F., Miyakawa, R., Chiba, A., Onodera, S., Hori, S., et al. (2018). Broad impact of extracellular DNA on biofilm formation by clinically isolated Methicillin-resistant and -sensitive strains of Staphylococcus aureus. Sci. Rep. 8:2254. doi: 10.1038/s41598-018-20485-Z

Tuchscherr, L., Korpos, E., van de Vyver, H., Findeisen, C., Kherkheulidze, S., Siegmund, A., et al. (2018). Staphylococcus aureus requires less virulence to establish an infection in diabetic hosts. Int. J. Med. Microbiol. 308, 761-769. doi: 10.1016/j.ijmm.2018.05.004

Vitko, N. P., Grosser, M. R., Khatri, D., Lance, T. R., and Richardson, A. R. (2016) Expanded Glucose Import Capability Affords Staphylococcus aureus Optimized Glycolytic Flux during Infection. mBio 7:e00296-16. doi: 10.1128/mBio.0029616

Vitko, N. P., Spahich, N. A., and Richardson, A. R. (2015). Glycolytic dependency of high-level nitric oxide resistance and virulence in Staphylococcus aureus. MBio 6:e00045-15. doi: 10.1128/mBio.00045-15

Waldrop, R., McLaren, A., Calara, F., and McLemore, R. (2014). Biofilm growth has a threshold response to glucose in vitro. Clin. Orthop. Relat. Res. 472, 3305-3310. doi: 10.1007/s11999-014-3538-5

Wu, J. A., Kusuma, C., Mond, J. J., and Kokai-Kun, J. F. (2003). Lysostaphin disrupts Staphylococcus aureus and Staphylococcus epidermidis biofilms on artificial surfaces. Antimicrob. Agents Chemother. 47, 3407-3414. doi: 10.1128/ aac.47.11.3407-3414.2003

Wu, X., Zha, J., Koffas, M. A. G., and Dordick, J. S. (2019). Reducing Staphylococcus aureus resistance to lysostaphin using CRISPR-dCas9. Biotechnol. Bioeng. 116, 3149-3159. doi: 10.1002/bit.27143

Yamada, S., and Matsumoto, A. (1986). Effect of mannitol and glucose on the distribution and trypsin susceptibility of protein A of Staphylococcus aureus. Microbiol. Immunol. 30, 993-1002. doi: 10.1111/j.1348-0421.1986.tb0 3029.x 
Yang, X. Y., Li, C. R., Lou, R. H., Wang, Y. M., Zhang, W. X., Chen, H. Z., et al. (2007). In vitro activity of recombinant lysostaphin against Staphylococcus aureus isolates from hospitals in Beijing, China. J. Med. Microbiol. 56(Pt 1), 71-76. doi: 10.1099/jmm.0.46788-0

Yi, Y., Shen, Y., Wu, Q., Rao, J., Guan, S., Rao, S., et al. (2018). Protective Effects of Oxymatrine on Vascular Endothelial Cells from High-Glucose-Induced Cytotoxicity by Inhibiting the Expression of A2B Receptor. Cell Physiol. Biochem. 45, 558-571. doi: 10.1159/000487033

Yin, S., Jiang, B., Huang, G., Gong, Y., You, B., Yang, Z., et al. (2017). Burn Serum Increases Staphylococcus aureus Biofilm Formation via Oxidative Stress. Front. Microbiol. 8:1191. doi: 10.3389/fmicb.2017.01191

You, Y., Xue, T., Cao, L., Zhao, L., Sun, H., and Sun, B. (2014). Staphylococcus aureus glucose-induced biofilm accessory proteins, GbaAB, influence biofilm formation in a PIA-dependent manner. Int. J. Med. Microbiol. 304, 603-612. doi: 10.1016/j.ijmm.2014.04.003

Conflict of Interest: The authors declare that the research was conducted in the absence of any commercial or financial relationships that could be construed as a potential conflict of interest.

Copyright (c) 2020 Luo, Yue, Chen, She, Wu and Wu. This is an open-access article distributed under the terms of the Creative Commons Attribution License (CC BY). The use, distribution or reproduction in other forums is permitted, provided the original author(s) and the copyright owner(s) are credited and that the original publication in this journal is cited, in accordance with accepted academic practice. No use, distribution or reproduction is permitted which does not comply with these terms. 